\title{
Component Analysis of Anthocyanins in Petals at Different Flowering Stages of Three Rosa rugosa Hybrid Cultivars
}

\author{
Mingyuan Zhao\#, Xiaoming Sui\#, Yu Wang, Zongda Xu, Xiaoyan Yu*, Xu Han* \\ College of Forestry, Shandong Agricultural University, Tai'an, China \\ Email: *yxyxst200402 14@163.com, *hanxusdau@163.com
}

How to cite this paper: Zhao, M.Y., Sui, X.M., Wang, Y., Xu, Z.D., Yu, X.Y. and Han, X. (2019) Component Analysis of Anthocyanins in Petals at Different Flowering Stages of Three Rosa rugosa Hybrid Cultivars. Advances in Bioscience and Biotechnology, 10, 1-11.

https://doi.org/10.4236/abb.2019.101001

Received: December 7, 2018

Accepted: January 5, 2019

Published: January 8, 2019

Copyright $\odot 2019$ by author(s) and Scientific Research Publishing Inc. This work is licensed under the Creative Commons Attribution International License (CC BY 4.0).

http://creativecommons.org/licenses/by/4.0/

\begin{abstract}
The species and contents of anthocyanins in plant petals can make plants appear pink, red, violet and blue, etc., and play a major role in the coloration of plants. In this study, the species and contents of anthocyanins in the petals of three $R$. rugosa hybrid cultivars, $R$. rugosa "Zizhi", $R$. rugosa "Fenzizhi" and $R$. rugosa "Baizizhi", were analyzed, and the direct cause of the differences in flower color of three $R$. rugosa hybrid cultivars was inferred. This paper provides a reference for the coloration mechanism and flower color breeding of $R$. rugosa. The specific methods are as follows: the petals of five flowering stages of three $R$. rugosa hybrid cultivars were used as materials, and the types and contents of anthocyanins contained in them were qualitatively and quantitatively analyzed by high performance liquid chromatography (HPLC). The same six kinds of anthocyanins were identified in $R$. rugosa "Zizhi" and $R$. rugosa "Fenzizhi", mainly based on the diglycoside of paeoniflorin and cyanidin. The relative contents of the two anthocyanins were higher at budding stage and initial opening stage. In the different flowering stages of $R$. rugosa "Zizhi", the content of Pn3G5G was up to $4280.84 \pm 20.82 \mu \mathrm{g} \cdot \mathrm{g}^{-1}$, and the content of Cy3G5G was up to $789.41 \pm 1.21 \mu \mathrm{g} \cdot \mathrm{g}^{-1}$. In $R$. rugosa "Fenzizhi", the highest content of Pn3G5G reached $1293.50 \pm 17.64 \mu \mathrm{g} \cdot \mathrm{g}^{-1}$, and the content of Cy3G5G was up to $358.86 \pm 3.94 \mu \mathrm{g} \cdot \mathrm{g}^{-1}$. It could be speculated that the difference in the contents of Pn3G5G and Cy3G5G was the main reason for the difference in coloration between the petals of $R$. rugosa "Zizhi" and $R$. rugosa "Fenzizhi". A total of five species of anthocyanins were identified in $R$. rugosa "Baizizhi" and their contents were relatively low. Compared with $R$. rugosa "Zizhi" and $R$. rugosa "Fenzizhi", the presence of Cy3G was not detected. Therefore, we speculated that the two reasons above might be responsible for the visual white flowers of $R$. rugosa "Baizizhi".
\end{abstract}

\#These authors contributed equally. 


\section{Keywords}

Rosa rugosa, Flower Color, Anthocyanin, HPLC

\section{Introduction}

Rosa rugosa is an important ornamental plant which belongs to the genus Rosa in the family Rosaceae. It is native to China and has a long history of cultivation. It is not only a precious Chinese herbal medicine, food and industrial raw materials, but also an excellent plant material for landscaping and soil and water conservation. The color of most Rosa rugosa varieties is purple, only a few varieties are pink and white, lacking yellow, orange and red flowers [1] [2]. The color of $R$. rugosa is very simple, which seriously affects its application in landscaping [3]. Therefore, understanding the mechanism of $R$. rugosa color development has become the goal of many researchers.

The flower color of the plant is related to the pigment type, the pigment content (including the relative content of various pigments), the internal or surface structure of the petals, but the most important factor is the types and contents of the anthocyanidin. Anthocyanin is one of the main pigments of plant petal colors and fruit colors. It is a kind of water-soluble pigment belonging to flavonoids. There are more than 20 kinds of anthocyanins, which are mainly found in plants, Cyanidin, Delphinidin, Pelargonidin, Petunidin, Peonidin, and Malvidin. Anthocyanins are found in vacuoles mainly in the form of glycosides in plants, often referred to as anthocyanins [4] [5]. Anthocyanins are formed by binding one or two sugar molecules at the B-ring C3 or A-ring C5 position of anthocyanin [6]. The species and contents of anthocyanins in plant petals can make plants appear pink, red, violet, blue, etc. Niu and Chu performed UV-visible scanning on the pigment in $R$. rugosa, and obtained two absorption peaks at 380 $\mathrm{nm}$ and $520 \mathrm{~nm}$, which were presumed to be anthocyanins [7]. Wang measured the anthocyanin species in Pingyin $R$. rugosa "Fenghua No. 1" and found that the main component was Cy3G5G [8]. Zhang et al. measured the pigment component of $R$. rugosa "Zizhi", and found that the diglucosides of Peonidin, Delphinidin, Cyanidin and Pelargonidin were the main components of $R$. rugosa "Zizhi" anthocyanins [9].

Studies have shown that anthocyanidin is extremely easy to extract and it is safe and non-toxic. At present, common methods for determining anthocyanin content include $\mathrm{pH}$ differential method [10], high performance liquid chromatography (HPLC) [11], high performance liquid chromatography-mass spectrometry (LC-MS) [12] and the like. However, the pH differential method is cumbersome, time consuming, and error-prone, and the LC-MS/MS method has higher sensitivity but higher cost. HPLC has the advantages of short time, high reproducibility and high analytical precision. It has been widely used in plants such as black rice [13], peony [14], blueberry [15], peanut [16], black soybean 
[17], cherry and cranberry [18].

In this study, HPLC was used to analyze the changes of the main components and contents of anthocyanins in three $R$. rugosa hybrid cultivars ( $R$. rugosa "Zizhi", $R$. rugosa "Fenizhi" and $R$. rugosa "Baizizhi") at five different flowering stages, and it provides a reference for the color development mechanism and color breeding of $R$. rugosa.

\section{Materials and Methods}

\subsection{Plant Materials}

With respect to Rosa, $R$. rugosa "Zizhi", $R$. rugosa "Fenizhi" and $R$. rugosa "Baizizhi" in the rose germplasm nursery of Shandong Agricultural University were used as test material. We collected petals at the budding stage, initial opening stage, half opening stage, full opening stage and wilting stage in the mornings of sunny days from 20 April to 10 May 2017. After flash freezing in liquid nitrogen, all samples, which were collected in triplicate, were put into a $-80^{\circ} \mathrm{C}$ refrigerator for storage.

\subsection{Total Anthocyanin Extraction and HPLC Analysis}

All samples $(0.1 \mathrm{~g}$ fresh weight) were homogenized in liquid nitrogen, after which they were extracted with $5 \mathrm{~mL}$ of an acidic methanol solution (70:0.1:29.9, $\left.\mathrm{v} / \mathrm{v} / \mathrm{v} ; \mathrm{CH}_{3} \mathrm{OH}: \mathrm{HCl}: \mathrm{H}_{2} \mathrm{O}\right)$ at $4{ }^{\circ} \mathrm{C}$ in darkness for $24 \mathrm{~h}$ and then sonicated for 30 min [19] [20]. After centrifugation, each extract was passed through a membrane filter $(0.22 \mathrm{~mm})$.

Qualitative and quantitative analyses of anthocyanins were performed via HPLC. The chromatographic analysis was conducted using a Prominence LC-20AT series HPLC system (Shimadzu, Inc., Japan) with a detection wavelength of $530 \mathrm{~nm}$, and the column (TC-C18 column, $5 \mu \mathrm{m}, 4.6 \mathrm{~mm} \times 250 \mathrm{~mm}$ ) was maintained at $30^{\circ} \mathrm{C}$. The eluent consisted of an aqueous solution A $(0.1 \%$ formic acid in water) and organic solvent $\mathrm{B}$ (acetonitrile). The gradient elution program was modified as described previously [21]: $0 \mathrm{~min}, 10 \% \mathrm{~B} ; 15 \mathrm{~min}, 17 \%$ B; $20 \mathrm{~min}, 23 \% \mathrm{~B} ; 25 \mathrm{~min}, 23 \% \mathrm{~B}$; and $30 \mathrm{~min}, 10 \% \mathrm{~B}$. Moreover, the eluent flow rate was $1.0 \mathrm{ml} / \mathrm{min}$, with a $10 \mu \mathrm{L}$ injection volume. Cy3G $\left(\mathrm{C}_{21} \mathrm{H}_{21} \mathrm{CLO}_{11}\right)$, Cy3G5G $\left(\mathrm{C}_{27} \mathrm{H}_{31} \mathrm{ClO}_{16}\right)$, Pg3G $\left(\mathrm{C}_{21} \mathrm{H}_{21} \mathrm{ClO}_{10}\right)$, Pg3G5G $\left(\mathrm{C}_{27} \mathrm{H}_{31} \mathrm{ClO}_{15}\right)$, Pn3G $\left(\mathrm{C}_{22} \mathrm{H}_{23} \mathrm{ClO}_{11}\right)$, Pn3G5G $\left(\mathrm{C}_{28} \mathrm{H}_{33} \mathrm{ClO}_{16}\right)$, Dp3G $\left(\mathrm{C}_{21} \mathrm{H}_{21} \mathrm{ClO}_{12}\right)$ and Dp3G5G $\left(\mathrm{C}_{27} \mathrm{H}_{31} \mathrm{ClO}_{17}\right)$ (EXTRASYNTHESE Trading Company, France) were used as references for anthocyanin analysis. Three independent biological replicates were measured for each sample.

\subsection{Statistical Analyses}

Three independent biological replicates were measured for each sample and the data presented as the mean \pm standard error (SE). Where applicable, data were analyzed by Student's $t$ test in a LSD analysis. Values of $\mathrm{P}<0.01$ were considered to be statistically significant. 


\section{Results}

\subsection{Analysis of Flower Phenotype of Three R. rugosa Hybrid Cultivars}

In the flower phenotypic display of $R$. rugosa hybrid cultivars, from top to bottom are R. rugosa "Zizhi" (Figure 1(a)) R. rugosa "Fenizhi" (Figure 1(b)) and $R$. rugosa "Baizizhi" (Figure $1(\mathrm{c})$ ). The color of $R$. rugosa "Zizhi" is purple and is the most common color. As the flowers gradually open, the color is slightly lighter and is the shallowest at the wilting stage. The color of $R$. rugosa "Fenizhi" is pink, and the color is the deepest at the initial opening stage. As the flowers gradually open, the color is slightly lighter, and the flower color is the shallowest at the wilting stage. The color of $R$. rugosa "Baizizhi" is white. In the budding stage and initial opening stage, its petals are slightly pinkish at the edge, and the pink disappears and turns white in the half opening stage.

\subsection{HPLC Determination of Petals in Five Flowering Stages of Three R. rugosa Hybrid Cultivars}

Qualitative and quantitative analysis of the anthocyanins detected by the $530 \mathrm{~nm}$ chromatogram was carried out in combination with the standard. Six kinds of anthocyanins were detected in $R$. rugosa "Zizhi" (Figure 2(a)), which were Cy3G5G, Cy3G, Pn3G5G, Pn3G, Pg3G5G and Pg3G. From the different flowering stages of $R$. rugosa "Zizhi" (Table 1), the content of Pn3G5G was the highest, the highest was $4280.84 \pm 20.82 \mu \mathrm{g} \cdot \mathrm{g}^{-1}$, and Pn3G5G accounted for $82.83 \%$ of the total anthocyanin contents in different flowering periods. The content of Cy3G5G was higher, the highest was $789.41 \pm 1.21 \mu \mathrm{g} \cdot \mathrm{g}^{-1}$, and Cy3G5G accounts for $13.56 \%$ of the total anthocyanin contents in different flowering periods. The change trend of the two main anthocyanin contents was budding stage > initial opening stage $>$ half opening stage $>$ full opening stage $>$ wilting stage. The contents of the other four anthocyanins were lower.

Six kinds of anthocyanins (Figure 2(b)) were detected in $R$. rugosa "Fenzizhi”, which were Cy3G5G, Cy3G, Pn3G5G, Pn3G, Pg3G5G, and Pg3G. From the different flowering stages of $R$. rugosa "Fenizhi" (Table 2), the content of Pn3G5G was the highest, the highest was $1293.50 \pm 17.64 \mu \mathrm{g} \cdot \mathrm{g}^{-1}$, and Pn3G5G accounted for $76.93 \%$ of the total anthocyanin contents in different flowering stages; The content of Cy3G5G was higher, the highest was $358.86 \pm 3.94 \mu \mathrm{g} \cdot \mathrm{g}^{-1}$, and Cy3G5G accounted for $20.03 \%$ of the total anthocyanin contents in different flowering stages. The change trend of the two main anthocyanin contents was initial opening stage $>$ half opening stage $>$ full opening stage $>$ budding stage $>$ wilting stage. The contents of the other four anthocyanins were lower.

Five kinds of anthocyanins (Figure 2(c)) were detected in R. rugosa "Baizizhi", which were Cy3G5G, Pn3G5G, Pn3G, Pg3G5G and Pg3G. From the different flowering stages of $R$. rugosa "Baizizhi" (Table 3), the anthocyanin contents in each period was low and basically stable. Among them, Cy3G5G and Pn3G5G reached maximum values in the full opening stage, which were $1.26 \pm 0.01 \mu \mathrm{g} \cdot \mathrm{g}^{-1}$ 


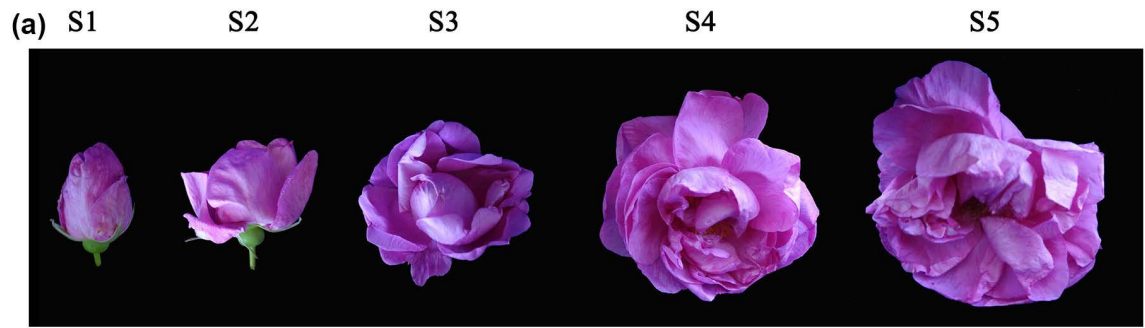

(b) $\mathrm{S} 1$

S2

S4 S5

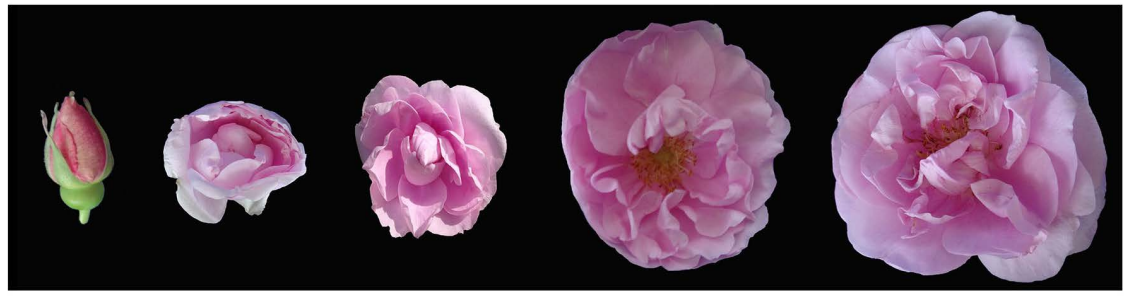

(c) S1

S2

S3

S4

S5

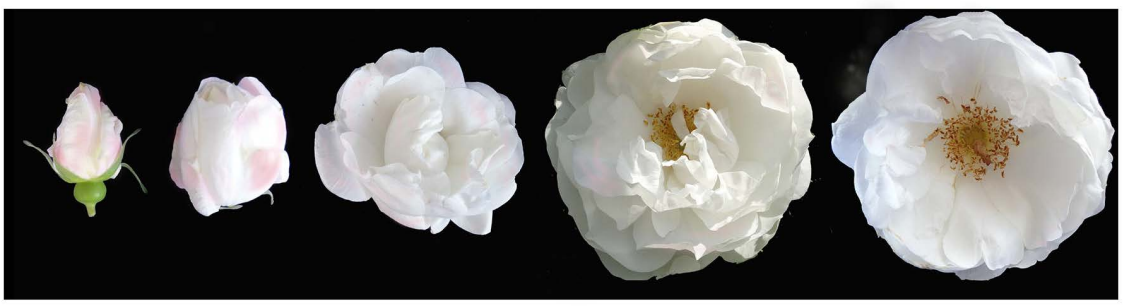

Figure 1. Flower phenotype of three R. rugosa hybrid cultivars. (a) Five different flowering stages of $R$. rugosa "Zizhi"; (b) Five different flowering stages of $R$. rugosa "Fenzizhi"; (c) Five different flowering stages of $R$. rugosa "Baizizhi". S1, budding stage; S2, initial opening stage; $S 3$, half opening stage; 4 , full opening stage; $S 5$, wilting stage.

and $3.39 \pm 0.01 \mu \mathrm{g} \cdot \mathrm{g}^{-1}$, respectively. Pg3G5G began to appear in the initial opening stage, and then its content dropped sharply, and it was not detected in the half opening stage. Pg $3 \mathrm{G}$ also appeared at the initial opening stage, after which the content remained basically stable.

Among the three $R$. rugosa varieties, six kinds of anthocyanins were detected in $R$. rugosa "Zizhi" and $R$. rugosa "Fenzizhi", and only five kinds were detected in $R$. rugosa "Baizizhi". Compared to the other two varieties, Cy3G was not detected in $R$. rugosa "Baizizhi". The results of the comparison of anthocyanin contents among different $R$. rugosa varieties in five flowering stages (Figure 3) are $R$. rugosa "Zizhi" $>R$. rugosa "Fenzizhi" $>R$. rugosa "Baizizhi". Compared with $R$. rugosa "Zizhi" and $R$. rugosa "Fenzizhi", the contents of anthocyanin in $R$. rugosa "Baizihi" was very low. In addition, the presence of two kinds of anthocyanins of delphinidin was not detected in any of the three varieties.

\section{Discussion}

$R$. rugosa "Zizhi" is selected from the hybrid offspring of $R$. rugosa "Jinyiyurui" and Rosa davarica [22]. Its shrubs are erect and open, with mauve branches. The prickles are erect and sparse. And it can be flowered many times a year. It is a 


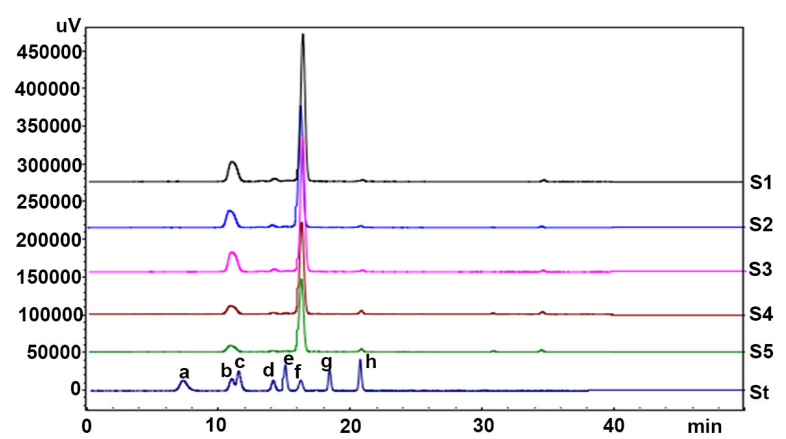

(a)

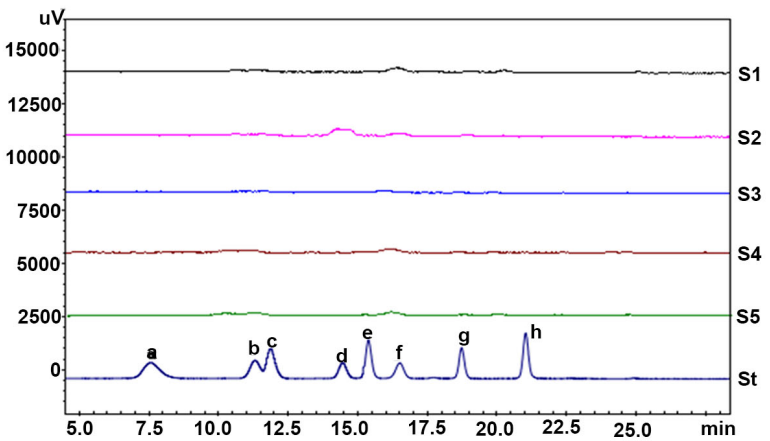

(c)

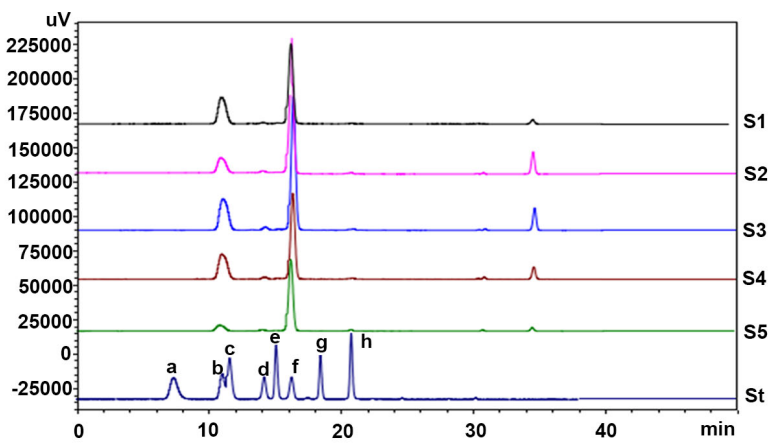

(b)

Figure 2. HPLC chromatograms in (a) R. rugosa "Zizhi", (b) R. rugosa "Fenzizhi" and (c) R. rugosa "Baizizhi". Eight kinds of anthocyanin standards (St.) were used for detection: (a) Dp3G5G; (b) Cy3G5G; (c) Dp3G; (d) Pg3G5G; (e) Cy3G; (f) Pn3G5G; (g) Pg3G; (h) Pn3G.

Table 1. Contents of anthocyanins in the flowers of $R$. rugosa "Zizhi” ( $\left.\mu \mathrm{g} \cdot \mathrm{g}^{-1} \mathrm{FW}\right)$.

\begin{tabular}{|c|c|c|c|c|c|c|c|c|c|}
\hline Name & Stage & Сy3G & Сy3G5G & Pn3G & Pn3G5G & $\mathrm{Pg} 3 \mathrm{G}$ & Pg3G5G & Dp3G & Dp3G5G \\
\hline \multirow{4}{*}{$\begin{array}{l}\text { R. rugosa } \\
\text { "Zizhi" }\end{array}$} & S1 & $28.27 \pm 0.16 \mathrm{D}$ & $789.74 \pm 1.21 \mathrm{~B}$ & $21.61 \pm 0.51 \mathrm{D}$ & $4280.84 \pm 20.82 \mathrm{~A}$ & $4.56 \pm 0.14 \mathrm{D}$ & $117.57 \pm 3.21 \mathrm{C}$ & - & - \\
\hline & S3 & $27.20 \pm 0.16 \mathrm{D}$ & $674.62 \pm 3.69 \mathrm{~B}$ & $24.09 \pm 0.01 \mathrm{D}$ & $3511.42 \pm 28.65 \mathrm{~A}$ & $4.54 \pm 0.17 \mathrm{D}$ & $88.31 \pm 0.70 \mathrm{C}$ & - & - \\
\hline & S4 & $22.88 \pm 0.08 \mathrm{D}$ & $334.67 \pm 2.57 \mathrm{~B}$ & $40.55 \pm 0.16 \mathrm{CD}$ & $2629.25 \pm 9.84 \mathrm{~A}$ & $2.98 \pm 0.01 \mathrm{E}$ & $55.34 \pm 0.80 \mathrm{C}$ & - & - \\
\hline & S5 & $16.85 \pm 0.03 \mathrm{E}$ & $251.62 \pm 2.96 \mathrm{~B}$ & $32.30 \pm 0.09 \mathrm{D}$ & $2091.76 \pm 5.04 \mathrm{~A}$ & $7.44 \pm 0.06 \mathrm{E}$ & $44.62 \pm 0.20 \mathrm{C}$ & - & - \\
\hline
\end{tabular}

${ }^{*}$ Data are the mean values \pm SE of three independent replicates. Different upper case letters represent significant difference which is calculated using LSD analysis at the level of $\mathrm{P}<0.01$. “-” means that no corresponding anthocyanin is detected.

species with very high ornamental value, and widely used in landscaping. $R$. rugosa "Fenzizhii" and $R$. rugosa "Baizizhi" are two bud varieties of $R$. rugosa "Zizhi", the former is pink, and the latter is white. Because most of the $R$. rugosa flowers are purple, this study used the universally representative $R$. rugosa "Zizhi", and then selected two different flower color bud varieties, studied their anthocyanin composition separately, and analyzed the causes of the difference in flower color.

For three $R$. rugosa hybrid cultivars, the color of $R$. rugosa "Zizhi" is the deepest and it's purple; the color of $R$. rugosa "Fenzizhi" is second and it's pink; the color of $R$. rugosa "Baizizhi" is the lightest and it's white. For example, Pn3G5G, 
Table 2. Contents of anthocyanins in the flowers of $R$. rugosa "Fenzizhi" ( $\left.\mu \mathrm{g} \cdot \mathrm{g}^{-1} \mathrm{FW}\right)$.

\begin{tabular}{|c|c|c|c|c|c|c|c|c|c|}
\hline Name & Stage & Сy3G & Cy3G5G & Pn3G & Pn3G5G & Pg3G & Pg3G5G & Dp3G & Dp3G5G \\
\hline \multirow{5}{*}{$\begin{array}{l}\text { R. rugosa } \\
\text { "Fenzizhi" }\end{array}$} & S1 & $8.77 \pm 0.10 \mathrm{C}$ & $358.86 \pm 3.94 \mathrm{~B}$ & $4.34 \pm 0.07 \mathrm{C}$ & $1293.50 \pm 17.64 \mathrm{~A}$ & $1.90 \pm 0.06 \mathrm{C}$ & $27.35 \pm 0.28 \mathrm{C}$ & - & - \\
\hline & S2 & $17.08 \pm 0.13 \mathrm{D}$ & $712.35 \pm 1.94 \mathrm{~B}$ & $13.11 \pm 0.12 \mathrm{D}$ & $2978.92 \pm 12.66 \mathrm{~A}$ & $2.53 \pm 0.05 \mathrm{D}$ & $90.63 \pm 1.05 \mathrm{C}$ & - & - \\
\hline & S3 & $11.70 \pm 0.26 \mathrm{D}$ & $651.82 \pm 4.25 \mathrm{~B}$ & $8.27 \pm 0.13 \mathrm{D}$ & $2094.54 \pm 5.23 \mathrm{~A}$ & $1.51 \pm 0.01 \mathrm{D}$ & $63.81 \pm 0.54 \mathrm{C}$ & - & - \\
\hline & S4 & $8.53 \pm 0.23 \mathrm{D}$ & $530.40 \pm 5.85 \mathrm{~B}$ & $7.17 \pm 0.10 \mathrm{D}$ & $1332.25 \pm 7.12 \mathrm{~A}$ & $1.47 \pm 0.02 \mathrm{D}$ & $43.11 \pm 0.82 \mathrm{C}$ & - & - \\
\hline & S5 & $5.38 \pm 0.08 \mathrm{DE}$ & $124.96 \pm 1.81 \mathrm{~B}$ & $9.49 \pm 0.01 \mathrm{D}$ & $1116.41 \pm 2.31 \mathrm{~A}$ & $0.80 \pm 0.01 \mathrm{E}$ & $26.41 \pm 0.27 \mathrm{C}$ & - & - \\
\hline
\end{tabular}

${ }^{*}$ Data are the mean values \pm SE of three independent replicates. Different upper case letters represent significant difference which is calculated using LSD analysis at the level of $\mathrm{P}<0.01$. "-" means that no corresponding anthocyanin is detected.

Table 3. Contents of anthocyanins in the flowers of $R$. rugosa "Baizizhi" ( $\left.\mu \mathrm{g} \cdot \mathrm{g}^{-1} \mathrm{FW}\right)$.

\begin{tabular}{cccccccccc}
\hline Name & Stage & Cy3G & Cy3G5G & Pn3G & Pn3G5G & Pg3G & Pg3G5G & Dp3G & Dp3G5G \\
\hline & S1 & - & $0.57 \pm 0.01 \mathrm{~B}$ & $0.38 \pm 0.01 \mathrm{D}$ & $2.36 \pm 0.02 \mathrm{~A}$ & - & - & - & - \\
& $\mathrm{S} 2$ & - & $0.82 \pm 0.01 \mathrm{C}$ & $0.23 \pm 0.01 \mathrm{E}$ & $2.02 \pm 0.01 \mathrm{~B}$ & $0.65 \pm 0.01 \mathrm{D}$ & $3.07 \pm 0.01 \mathrm{~A}$ & - & - \\
$\begin{array}{c}\text { R. rugosa } \\
\text { "Baizizhi" }\end{array}$ & $\mathrm{S} 3$ & - & $0.52 \pm 0.01 \mathrm{~B}$ & $0.16 \pm 0.01 \mathrm{E}$ & $1.43 \pm 0.01 \mathrm{~A}$ & $0.23 \pm 0.01 \mathrm{D}$ & - & - & - \\
& S4 & - & $1.26 \pm 0.01 \mathrm{~B}$ & - & $3.39 \pm 0.01 \mathrm{~A}$ & $0.27 \pm 0.01 \mathrm{C}$ & - & - & - \\
& S5 & - & - & - & $2.54 \pm 0.01 \mathrm{~A}$ & $0.56 \pm 0.01 \mathrm{C}$ & - & - & - \\
\hline
\end{tabular}

${ }^{*}$ Data are the mean values \pm SE of three independent replicates. Different upper case letters represent significant difference which is calculated using LSD analysis at the level of $\mathrm{P}<0.01$. "-" means that no corresponding anthocyanin is detected.

which was dominant in anthocyanin contents in three $R$. rugosa hybrid cultivars, had the highest content in $R$. rugosa "Zizhi", which was up to $4280.84 \pm 20.82$ $\mu \mathrm{g} \cdot \mathrm{g}^{-1}$; the highest content in $R$. rugosa "Fenzizhi" was $1293.50 \pm 17.64 \mu \mathrm{g} \cdot \mathrm{g}^{-1}$, and the highest content in $R$. rugosa "Baizizhi" was only $3.39 \pm 0.01 \mu \mathrm{g} \cdot \mathrm{g}^{-1}$. It can be seen that the anthocyanin contents are positively correlated with the flower color, and the deeper the flower color, the more the anthocyanin contents. In addition to the difference in anthocyanin species, Cy3G was not detected in $R$. rugosa "Baizizhi", suggesting that these two factors may be the cause of the color of $R$. rugosa "Baizizhi". Cy3G is a precursor of the synthesis of Cy3G5. Cy3G5G can be detected in $R$. rugosa "Baizizhi", but Cy3G cannot be detected. It can be speculated that Cy3G in $R$. rugosa "Baizizhi" is depleted before the flower bud stage and converted into Cy3G5G. Zhang et al. detected delphinidin in $R$. rugosa "Zizhi" and found that its content was basically stable [9]. However, we did not detect the presence of delphinidin in the HPLC test of anthocyanin species in five flowering stages of three $R$. rugosa varieties, which was inconsistent with her research results.

The types and contents of the pigments contained in the petals and the $\mathrm{pH}$ of the cell fluid are the main factors affecting the color of the flowers [23]. This study found that the anthocyanin derivatives in the three $R$. rugosa petals are mainly diglycoside, which indicated that the structure of the diglycoside anthocyanins in the three $R$. rugosa hybrid cultivars was very stable, which was consistent with previous reports [24] [25]. In addition, this study found that $R$. 


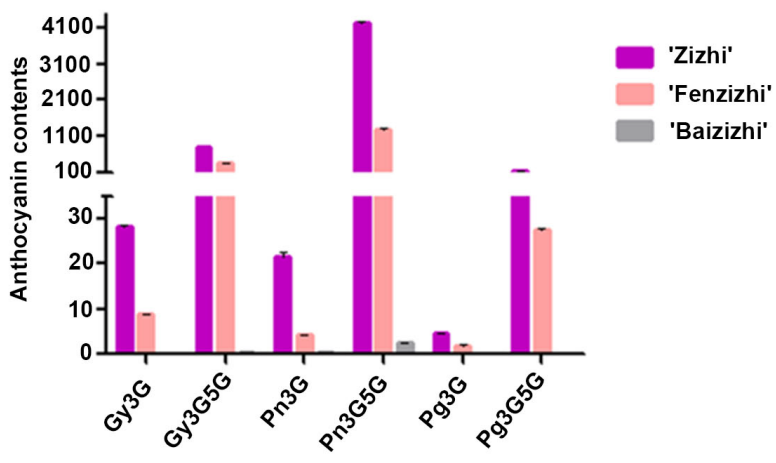

(a)

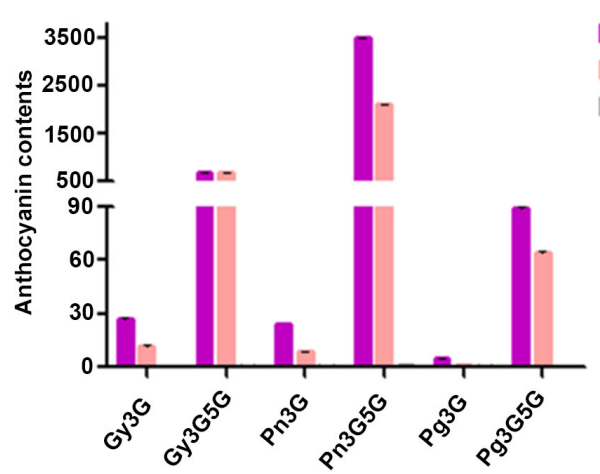

(c)

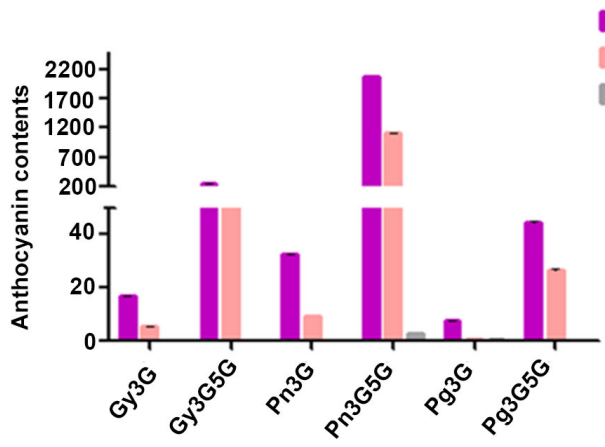

(e)
'Zizhi'

'Fenzizhi'

'Baizizhi'

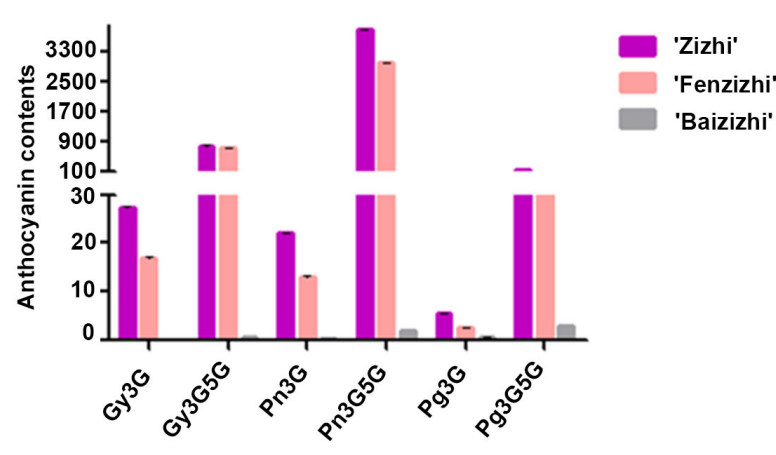

(b)

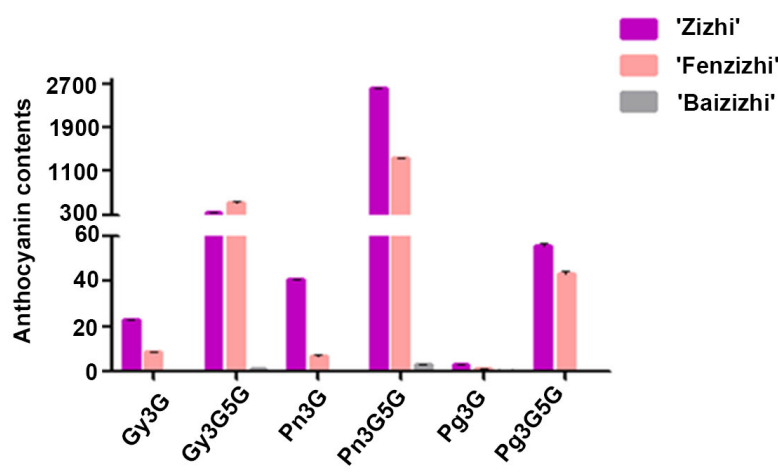

(d)

Figure 3. Comparison of anthocyanin contents of three $R$. rugosa hybrid cultivars at five flowering stages. (a) Budding stage; (b) Initial opening stage; (c) Half opening stage; (d) Full opening stage; (e) Wilting stage. The error bars represent the SDs of triplicate reactions. The experiment was repeated three times, and each yielded similar results.

rugosa "Zizhi" and R. rugosa "Fenzizhi" contain derivatives of paeoniflorin and cyanidin. In the different flowering stages of $R$. rugosa "Zizhi", the contents of Pn3G5G and Cy3G5G decreased with the opening of the petals. In the different flowering stages of $R$. rugosa "Fenzizhi", the contents of Pn3G5G and Cy3G5G also changed with the color of the flowers, reached the highest value in the initial opening period, and then gradually became lower. From this, it can be inferred that the flower color changes of $R$. rugosa "Zizhi" and $R$. rugosa "Fenzizhi" are 
mainly related to the changes of Pn3G5G and Cy3G5G contents.

Quantitative results of anthocyanins in petals of $R$. rugosa "Zizhi" and $R$. rugosa "Fenizhi" showed that most of the anthocyanins had the highest relative contents in the flower bud stage and the initial stage, and it can be inferred that most of the anthocyanins required of $R$. rugosa colors have been fully accumulated in the flower bud stage and the initial stage, and gradually degraded. With the opening of flowers in $R$. rugosa "Zizhi" and $R$. rugosa "Fenzizhi", the contents of anthocyanins decreased with the opening of the flowers. This may be as the flowers are opened, the petals gradually expand, and the light-receiving area of the petals increases, and the light affects its stability, causing it to gradually degrade, resulting in a gradual decrease in contents. Quantitative results of anthocyanins in petals of $R$. rugosa "Baizizhi" showed that Cy3G5G and Pn3G5G had been synthesized at the flower bud stage and reached the highest value in the flowering stage. It can be speculated that these two anthocyanins may be the main causes of the formation of $R$. rugosa "Baizizhi". Two kinds of anthocyanins, Pg3G5G and Pg3G, were synthesized in the initial stage. Pg3G5G was rapidly depleted after synthesis and could not be detected in the half-opening stage, which may be related to the pink disappearance of the petals edge at the half-opening stage of $R$. rugosa "Baizizhi". It can be speculated that Pg3G5G is involved in the formation of the pink edge of its petals.

\section{Conclusion}

In conclusion, in this study, we analyze the differences in the types and contents of anthocyanins in different flowering stages of three $R$. rugosa hybrid cultivars, speculating on the low anthocyanin contents and the lack of Cy3G, which may be the reasons for flower color generation of $R$. rugosa "Baizizhi". In addition, it is presumed that the difference in the contents of Pn3G5G and Cy3G5G is the main cause of colors between $R$. rugosa "Zizhi" and $R$. rugosa "Fenzizhi". Through the above analysis, we have preliminarily identified the direct causes of the differences in the color in three $R$. rugosa hybrid cultivars ( $R$. rugosa "Zizhi", $R$. rugosa "Fenzizhi" and $R$. rugosa "Baizizhi"), and we hope this will provide necessary information for subsequent color-improvement projects in $R$. rugosa.

\section{Conflicts of Interest}

The authors declare no conflicts of interest regarding the publication of this paper.

\section{References}

[1] Mikanagi, Y., Saito, N., Yokoi, M. and Tatsuzawa, F. (2000) Anthocyanins in Flowers of Genus Rosa, Sections Cinnamomeae (=Rosa), Chinenses, Gallicanae and Some Modern Garden Roses. Biochemical Systematics and Ecology, 28, 887-902. https://doi.org/10.1016/S0305-1978(99)00127-1

[2] Xu, Z.-D., Zhao, L.-Y., Zhang, L. and Yang, Z.Y. (2011) Analysis of Genetic Diversity and Construction of Fingerprint of Rosa rugosa by Srap. Scientia Agricultura 
Sinica, 44, 1662-1669.

[3] Yu, X.Y., Xing, S.T. and Zhao, L.Y. (2014) Analysis on the Barriers of Interspecific Hybridization between Rosa rugosa and Rosa rugosa Hybrid Cultivars. Scientia Agricultura Sinica, 47, 3112-3120.

[4] Grotewold, E. (2006) The Genetics and Biochemistry of Floral Pigments. Annual Review of Plant Biology, 57, 761-780. https://doi.org/10.1146/annurev.arplant.57.032905.105248

[5] Cuilian, G.E. (2012) Research on Anthocyanins Biosynthesis in Fruit. Acta Horticulturae Sinica, 39, 1655-1664.

[6] Clifford, M.N. and Scalbert, A. (2000) Ellagitannins-Nature, Occurrence and Dietary Burden. Journal of the Science of Food \& Agriculture, 80, 1118-1125. https://doi.org/10.1002/(SICI)1097-0010(20000515)80:7<1118::AID-JSFA570>3.0.C O;2-9

[7] Niu, J.Sh. and Chu, H.T. (1996) Study on the Properties, Extraction and Application of Edible Natural Rosa rugosa Pigment. Food Industry Science and Technology, 2, 24-26.

[8] Wang, B.X. (2010) Study on Analytical Method of Total Flavonoids in Rosa rugosa. Shandong University of Traditional Chinese Medicine, Jinan.

[9] Zhang, L., Xu, Z.D., Zhao, L.Y., et al. (2015) Analysis of Anthocyanin-Related Compounds and Metabolic Pathways in the Flowering Process of Rosa rugosa "Zizhi”. Scientia Agricultura Sinica, 48, 235-236.

[10] Sang, G.L., Vance, T.M., Nam, T.-G., et al. (2016) Evaluation of PH Differential and HPLC Methods Expressed as Cyanidin-3-Glucoside Equivalent for Measuring the Total Anthocyanin Contents of Berries. Journal of Food Measurement \& Characterization, 10, 562-568. https://doi.org/10.1007/s11694-016-9337-9

[11] Benvenuti, S., Brighenti, V. and Pellati, F. (2018) High-Performance Liquid Chromatography for the Analytical Characterization of Anthocyanins in Vaccinium myrtillus 1. (Bilberry) Fruit and Food Products. Analytical \& Bioanalytical Chemistry, 410, 3559-3571. https://doi.org/10.1007/s00216-018-0915-Z

[12] Bao, T., Xu, Y., Gowd, V., et al. (2016) Systematic Study on Phytochemicals and Antioxidant Activity of Some New and Common Mulberry Cultivars in China. Journal of Functional Foods, 25, 537-547. https://doi.org/10.1016/j.jff.2016.07.001

[13] Xia, X.D., Ling, W.H., Zheng, L., et al. (2006) Determination of Anthocyanin Component and Content in Black Rice Extract by HPLC. Food Science, 27, 206-208.

[14] Zhou, C.-H., Lu, S.-S., Zhang, Y., Hu, Y. and Tao, J. (2011) Analysis of Oleanolic and Ursolic Acids in Peony (Paeonia lactiflora pall.) by HPLC. Food Science, 32, 265-268.

[15] Wang, J., Kalt, W. and Sporns, P. (2000) Comparison between HPLC and MALDI-TOF MS Analysis of Anthocyanins in Highbush Blueberries. Journal of Agricultural \& Food Chemistry, 48, 3330-3335. https://doi.org/10.1021/jf000101g

[16] Asis, R. and Di, P.R.M. (2002) Determination of Aflatoxin b-1 in Highly Contaminated Peanut Samples Using HPLC and ELISA. Food \& Agricultural Immunology, 14, 201-208. https://doi.org/10.1080/09540100220145000

[17] Liu, Z.Y., Liu, X.Q., Bai, S.F., et al. (2011) Hplc Determination of Cyanidin-3-o-glucoside in Black Soybean Hulls Grown in Different Regions of China. Food Science, 32, 256-259.

[18] Karaaslan, N.M. and Yaman, M. (2016) Determination of Anthocyanins in Cherry and Cranberry by High-Performance Liquid Chromatography-Electrospray Ioni- 
zation-Mass Spectrometry. European Food Research \& Technology, 242, 127-135. https://doi.org/10.1007/s00217-015-2524-9

[19] Yang, Q., Yuan, T. and Sun, X.B. (2015) Preliminary Studies on the Changes of Flower Color during the Flowering Period in Two Tree Peony Cultivars. Acta Horticulturae Sinica, 42, 930-938.

[20] Sui, X.M., Zhao, M.Y., Han, X., Zhao, L.Y. and Xu, Z.D. (2019) RrGT1, a Key Gene Associated with Anthocyanin Biosynthesis, Was Isolated from Rosa rugosa and Identified via Overexpression and VIGS. Plant Physiology and Biochemistry, 135, 19-29. https://doi.org/10.1016/j.plaphy.2018.11.022

[21] Xie, D.Y., Sharma, S.B., Wright, E., Wang, Z.-Y. and Dixon, R.A. (2006) Metabolic Engineering of Proanthocyanidins through Co-Expression of Anthocyanidin Reductase and the PAP1 MYB Transcription Factor. The Plant Journal, 45, 895-907. https://doi.org/10.1111/j.1365-313X.2006.02655.x

[22] Li, Y.S. (2006) Studies on Germplasm Resources and Cultivars Classification of Rosa rugosa in China. Beijing Forestry University, Beijing.

[23] Sun, W., Li, C.-H., Wang, L.S. and Dai, S.L. (2010) Accumulation and Variation of Anthocyanins in Blue Flowers of Senecio Cruentus at Different Flowering Stages. Journal of Beijing Forestry University, 32, 128-134.

[24] Vyas, D., Sood, S. and Nagar, P.K. (2006) Physiological and Biochemical Studies during Flower Development in Two Rose Species. Scientia Horticulturae, 108, 390-396.

[25] Mori, M., Kondo, T. and Yoshida, K. (2009) Blue Flower Color Development by Anthocyanins: from Chemical Structure to Cell Physiology. Cheminform, 26, 884-915. 